\title{
Investigating The Structural Dynamics of the Water and Proton Channels Using snap-shots of Photosystem II
}

\author{
Mohamed Ibrahim \\ HU-Berlin, Berlin, Germany \\ mohamed.ibrahim@hu-berlin.de
}

\begin{abstract}
The water oxidation process in Photosystem II, a Bio-machinery that evolved nearly three billion years ago, fascinates us with its capabilities of harvesting solar energy and storing it in a chemical form. The X-ray Free Electron Lasers enabled us to study this phenomenal protein in ways that were not possible before. In the current manuscript, we introduce new approaches for XFEL data to understand better the catalytic activity, not only for PSII, via deeper analysis of the water network around the active site. Using a highresolution $1.89 \AA$ room temperature crystal structure of PS II and the re-processed crystallography data at various time points between the S2 to S3 transition of Kok's cycle, we identified the substrate water intake channel extends starting near O1 of the OEC to the lumenal side of the membrane. Three main well-coordinated structural events during the S2 to S3 transition occurred within the water channels, resulting in substrate insertion and proton egress. In particular, the rotation of D1-E65 and the appearance of new water before the substrate insertion likely facilitate proton removal through the $\mathrm{Cl} 1$ channel. While the arrival of new water near D1-E329 after the substrate insertion probably indicates the delivery via the $\mathrm{O} 1$ channel.
\end{abstract}

Keywords: XFEL, Photosystem II, Water Oxidation 